SSPE has been reported in vaccinated children without prior history of measles infection.

Materials and Methods: A retrospective study was done to analyze vaccinated children with SSPE without prior history of measles infection.

Results: Fifteen out of 22 cases of SSPE in the past 10 years were immunized for measles. Mean age of onset was 8.4 years with $80 \%$ of children being under 10 years of age. Average time of progression to advanced stage of the disease was 2.5 months -3 months (range: 15 days -5 months).

Discussion In vaccinated children, a subclinical or unrecognized measles infection is speculated to be the source of wild virus, with exposure occurring before vaccination. Earlier the age of exposure, higher is the risk of SSPE. Although thought to be slowly progressive, recent trend indicates a younger age of onset and faster progression of SSPE from onset to critically disabling stages. Vaccination with measles at 9 months changes the epidemiology of SSPE leaving younger infants susceptible to measles. Also, as per previous studies, maternally acquired antibodies probably wane off by 3 to 4 months. With current immunization schedule of administering first dose of measles vaccine at 9 months, children less than 9 months remain susceptible to measles and later SSPE. However, vaccination before 9 months is found to be both effective and safe.

Conclusion: This study proposes a relook at the need of reducing the age of vaccination against measles to 6 months.

\section{A0012: Network Changes in Eating Epilepsy: An MEG Study} Bhargava G.K., ${ }^{1,2}$ Mariyappa N., ${ }^{1}$ Shishir D., ${ }^{2}$ Raghavendra K., ${ }^{2}$ Ravindranadh C.M., ${ }^{2}$ Bharath R.D., ${ }^{3}$ Saini J.S., ${ }^{3}$

Sanjib Sinha ${ }^{1,2}$

${ }^{1}$ MEG Research Lab, National Institute of Mental Health and Neurosciences, Bengaluru, Karnataka, India

2Department of Neurology, National Institute of Mental Health and Neurosciences, Bengaluru, Karnataka, India

${ }^{3}$ Department of Neuroimaging and Interventional Radiology, National Institute of Mental Health and Neurosciences, Bengaluru, Karnataka, India

Objective: To examine the network differences in eating epilepsy at resting state and during eating period using MEG recording.

Methodology Resting state MEG data were analyzed in 17 patients with eating epilepsy $(\mathrm{M}: \mathrm{F}=12: 5$, age at onset $=9.66 \pm 8.63$ years, age at evaluation $=20.6 \pm 8.7$ years $)$. All underwent presurgical workup including MRI, EEG, video-EEG, MEG, and neuropsychological assessment. MEG recording while eating (rice) was obtained in five patients $(\mathrm{M}: \mathrm{F}=2: 3)$. MRI (brain) showed normal-9; left perisylvian gliosis-4, bilateral peri-sylvian gliosis-2, left MTS-1, and left temporal astrocytoma-1. Data were down sampled to $500 \mathrm{~Hz}$, artifacts removed and bandpass filtered between 0.5 and 150 $\mathrm{Hz}$. Data were transformed to source space by beam forming and parcellated. Amplitude envelope correlation was applied to six frequency bands (delta: $1-4 \mathrm{~Hz}$, theta: 5-7 Hz, $\sigma: 8-12$ $\mathrm{Hz}, \beta$ : 13-30 Hz, gamma1: 30-60 Hz, gamma 2: 60-90 Hz) to derive adjacency matrices for both conditions. Network measures (clustering coefficient, modularity, local efficiency, betweenness centrality, degree, and path length) were derived using graph theory. Group comparisons were FDR corrected.

Results: Resting state networks showed increased clustering and pathological local information processing in the superior, inferior, and medial parietal regions and prefrontal regions predominantly in the $\beta$ and gamma frequency bands $(p<0.036)$. While eating, network changes involved the precuneus, inferior parietal, right prefrontal, bilateral calcarine, and entorhinal regions in the $\beta$ and gamma bands $(p<0.027)$.

Conclusion: Previous electrophysiological and functional imaging studies have implicated involvement of perisylvian region. Pathological information processing was noted across the perisylvian regions dominantly on the right hemisphere in this study. Eating resulted in recruitment of bilateral hubs between the parietal, temporal, and occipital structures.

\section{A0013: Impact of Epileptic Seizures in the Neurological Intensive Care Unit (NICU) on Glasgow Coma Scale (GCS) Singh G., ${ }^{1}$ Paul B.S., ${ }^{1}$ Chaudhary A.K., ${ }^{1}$ Singh G. ${ }^{1}$ \\ 'Dayanand Medical College and Hospital, Ludhiana, Punjab, India}

Aims: To determine the frequency, magnitude, range, and duration of change in GCS in the aftermath of seizures occurring in the neurological intensive care unit.

Methods: Subjects were enrolled if they had a seizure during admission to a neurological intensive care unit. Their last recorded GCS prior to the seizures was extracted from chart review and GCS monitored prospectively at 2, 4, 8, 24, and 48 hours after the seizure. For comparison, subjects with matching GCS scores were also monitored for 48 hours to document fluctuations in their scores.

Results: Mean preseizure GCS in cases $(n=50)$ was $(10 \pm 4)$ and was similar to controls $(n=50)(10 \pm 4)$. Immediately after the seizure, the mean GCS fell to (6 \pm 3$)$ and thereafter increased progressively at 2 hours $(8 \pm 4), 4$ hours ( $9 \pm$ 4), 8 hours ( $9 \pm 4$.), 12 hours ( $9 \pm 5$ ), 24 hours ( $9 \pm 5$ ), and 48 hours $(9 \pm 5)$. However, the mean GCS did not return to preseizure values even after 48 hours. No deviations were noted in the control group. At the end of 2 hours, 37 cases still had deranged GCS; end of 4 hours, 26 patients had deranged GCS; at 8 hours, 18 patients had deranged GCS; at 12 hours and 24 hours, 16 patients; and finally, at 48 hours, 13 patients still had deranged GCS.

Conclusion: Seizures in the ICU lead to drop in GCS of those affected and this drop may persist even up to 48 hours. This may confound the assessment of GCS. 